\title{
MAXIMAL IDEALS IN POLYNOMIAL RINGS
}

\author{
ANTHONY V. GERAMITA ${ }^{1}$
}

\begin{abstract}
We show that if $R$ is a regular local ring of dimension 2 and $A=R[X]$ then every maximal ideal of $A$ is an ideal-theoretic complete intersection.
\end{abstract}

The purpose of this short note is to provide some additional evidence for one of the conjectures of Eisenbud and Evans [2]. We show that if $R$ is a regular local ring of dimension two and $A=R[X]$ is a polynomial ring in the indeterminate $X$ then every maximal ideal of $A$ may be generated by a set of elements whose cardinality is equal to the height of the maximal ideal, i.e. every maximal ideal is an ideal-theoretic complete intersection.

\section{General remarks.}

Definition.. If $R$ is a ring and $M$ is a finitely generated $R$-module, we let $\nu(M)=$ least number of elements of $M$ needed to generate $M$.

The first theorem we state gives a lower bound on $v(M)$.

Theorem A (Krull's Hauptidealsatz). If $R$ is a noetherian ring and $I$ is an ideal of $R$ then height $I=\mathrm{ht} I \leqq v(M)$.

The next two theorems will be used in what follows so we shall state them for easy reference.

THEOREM B. If $R$ is a noetherian ring and $M$ a maximal ideal in $R\left[X_{1}, \cdots, X_{n}\right]$ and $M \cap R=p$ then $p$ is a prime ideal of $R$ and ht $M=$ ht $p+$ $n$.

Proof. [4, Theorem 149].

THEOREM C. If $R$ is a noetherian ring and $M \subset R\left[X_{1}, \cdots, X_{n}\right]$ is maximal and $p=M \cap R$ then $R / p$ is a semilocal ring of Krull dimension $\leqq 1$.

ProOF. [1].

Received by the editors February 13, 1973.

AMS (MOS) subject classifications (1970). Primary 13A15, 13B25, 13C10, 13C15, 13H05, 14M10.

Key words and phrases. Number of generators, polynomial rings, maximal ideals, projective modules.

${ }_{1}^{1}$ This work was supported in part by the National Research Council of Canada under Grant A8488.

(c) American Mathematical Society 1973 
Thus, Theorem $\mathrm{C}$ says that the prime ideals of $R$ that are the contractions of maximal ideals in $R\left[X_{1}, \cdots, X_{n}\right]$ are either maximal or are contained in only finitely many prime ideals.

2. The main theorem. From now on $R$ will denote a regular local ring of dimension two and we shall be considering maximal ideals in $A=R[X]$. We first note that for those maximal ideals $M$ of $A$ which contract to the maximal ideal of $R$ the problem of determining $v(M)$ is trivial. By Theorem A above $v(M) \geqq 3$ and it is clear that $M$ may be generated by the two elements which generate the maximal ideal $m$ of $R$ and a generator for the principal ideal $M / m A$. Thus $v(M)=3$.

We need then only consider maximal ideals $M$ of $A$ such that $M \cap R=p$ and $p$ not maximal in $R$.

From Theorem C we see that ht $p=1$ and hence from Theorem B that ht $M=2$. Our theorem will follow as a corollary to the following adaptation of an idea of $\mathrm{M}$. Murthy [6].

THEOREM 1. Let $R$ be a regular integral domain and suppose that all projective $R$-modules of rank two are free. Let $M$ be a maximal ideal of $R$ such that ht $M=2$. Then $v(M)=2$.

Proof. If $M$ is maximal and ht $M=2$ then the global dimension $R_{M}=2$ and the homological dimension of $R / M$ (as an $R$-module) is $=2$. Thus, the homological dimension of $M$ (as an $R$-module) is $=1$.

Since $0 \rightarrow M \rightarrow R \rightarrow R / M \rightarrow 0$ is an exact sequence of $R$-modules we have

$$
\operatorname{Ext}^{1}(M, R) \simeq \operatorname{Ext}^{2}(R / M, R) \simeq \operatorname{Ext}_{R_{M}}^{2}\left(R_{M} / M R_{M}, R_{M}\right) .
$$

Since $R_{M}$ is regular local of dimension 2 and $M R_{M}$ is generated by an $R_{M}$-sequence of length 2 we have

$$
\operatorname{Ext}_{R_{M}}^{2}\left(R_{M} / M R_{M}, R_{M}\right) \simeq \operatorname{Hom}_{R_{M}}\left(R_{M} / M R_{M}, R_{M} / M R_{. M}\right)
$$

(see e.g., Kaplansky [4, pp. 100-101]).

But $\operatorname{Hom}_{R_{M}}\left(R_{M} / M R_{M}, R_{M} / M R_{M}\right) \simeq R_{M} / M R_{M} \simeq R / M$, this last isomorphism arising from the fact that $M$ is maximal.

Thus, $\operatorname{Ext}^{1}(M, R)$ is a cyclic $R$-module. By a lemma of Serre [7] this means that there is projective extension of $M$ by $R$, i.e., a short exact sequence $0 \rightarrow R \rightarrow P \rightarrow M \rightarrow 0$ where $P$ is a projective $R$-module. $P$ must necessarily have rank $=2$ and so by hypothesis $P$ is free. Since $M$ is a homomorphic image of a 2 -generator module, $v(M) \leqq 2$. Since ht $M=2$ we have $v(M)=2$.

COROLLARY. If $R$ is a regular local ring of dimension two and $A=R[X]$, then $v(M)=$ ht $M, \forall$ maximal ideals $M$ of $A$.

Proof. If ht $M=3$ the remarks preceding Theorem 1 are in force. 
If ht $M=2$, we notice that if $A$ is as above then Murthy has shown [5] that all finitely generated projective $A$-modules are free. Thus the theorem is applicable in this case and we conclude that $\nu(M)=2$.

3. Further remarks. The question, does $v(M)=$ ht $M$ for maximal ideals $M$ in $A=R\left[X_{1}, \cdots, X_{n}\right]$ where $R$ is regular local of any dimension, is an obvious one to ask. I do not know if the question has an affirmative answer. It is worth noting that Endo has shown that the question has an affirmative answer if $R$ has dimension one [3].

\section{BIBLIOGRAPHY}

1. E. Artin and J. Tate, A note on finite ring extensions, J. Math. Soc. Japan 3 (1951), 74-77. MR 13, 427.

2. D. Eisenbud and E. G. Evans, Three conjectures about modules over polynomial rings, Conference on Commutative Algebra (Lawrence, Kansas 1972), Lecture Notes in Math., vol. 311, Springer-Verlag, Berlin and New York.

3. S. Endo, Projective modules over polynomial rings, J. Math. Soc. Japan 15 (1963), 339-352. MR 27 \#5808.

4. I. Kaplansky, Commutative rings, Allyn and Bacon, Boston, Mass., 1970. MR 40 \#7234.

5. M. P. Murthy, Projective A[X]-modules, J. London Math. Soc. 41 (1966), 453-456. MR 34 \#188.

6. - Vector bundles over affine surfaces birationally equivalent to a ruled surface, Ann. of Math. (2) 89 (1969), 242-253. MR 39 \#2774.

7. J.-P. Serre, Sur les modules projectifs, Sém. Dubreil-Pisot 1960/61, no. 2.

Department of Mathematics, Queen's University, Kingston, Ontario, Canada 